\title{
Is APOC3 the driver of cardiovascular disease in people with type I diabetes mellitus?
}

\author{
Henry N. Ginsberg and Gissette Reyes-Soffer \\ Division of Preventive Medicine and Nutrition, Department of Medicine, Vagelos College of Physicians and Surgeons of Columbia University, New York, New York, USA.
}

\begin{abstract}
In this issue of the $J C I$, Kanter et al. make a strong case for implicating apolipoprotein C3 (APOC3) as a central player in atherosclerotic cardiovascular disease that is commonly seen in individuals with type 1 diabetes mellitus (T1DM). Kanter and colleagues suggest that insulin deficiency elevates plasma APOC3 as well as atherogenic triglyceriderich (TG-rich) lipoproteins (TRLs). Using two mouse models of T1DM, the authors investigated APOC3-mediated inhibition of both TC hydrolysis by lipoprotein lipase and hepatic uptake of remnant lipoproteins. They suggest that poorly catabolized lipoproteins, enriched in both APOC3 and APOE content, are particularly atherogenic. Notably, treating both mouse models with an APOC3 antisense oligonucleotide lowered both plasma APOC3 and TRLs, and prevented atherosclerosis. These impactful mouse studies were supported by the initial finding that APOC3 predicted coronary artery disease events in participants of the prospective Coronary Artery Calcification in Type 1 Diabetes study with normal TG levels.
\end{abstract}

Teasing apart the predictive value of TG and APOC3

The Coronary Artery Calcification in Type 1 Diabetes (CACTI) study was a cross-sectional cohort study designed to evaluate whether insulin resistance and/or insulin resistance-related factors are linked to calcification of the coronary arteries in people with type 1 diabetes mellitus (T1DM) (1). Kanter et al. determined the power of APOC3 and triglyceride (TG) as predictors of future coronary artery disease (CAD) events in a case-cohort subpopulation from CACTI (2). However, even well-designed, prospective observational studies can establish only correlation, not causality. Table 1 in the study by Kanter et al. (2) is a clear example of the difficulties investigators face in such studies: the study participants were older, characterized by longer duration of diabetes; higher blood pres- sure; higher plasma levels of TG, LDL cholesterol, and HbA1C; higher rates of smoking; and lower estimated glomerular filtration rate (eGFR). Although TG and APOC3 both predicted CAD events in unadjusted models, their power was not significantly different from each other. Adjustment of models 1, 2, and 3, for increasing numbers of the covariates listed above and omission of TG, resulted in APOC3 remaining a strong and significant predictor of patient outcomes. However, in model 4, which included all of the significant covariates used in model 3, as well as TG, APOC3 lost its predictive power, revealing the difficulty in teasing apart the relative contributions of TG and APO3C to atherosclerotic disease. Kanter et al. further interrogated the roles of each biomarker by adding TG to a multivariate model that included APOC3 and vice versa, and the results suggest-

\section{- Related Article: p. 4165}

Conflict of interest: The authors have declared that no conflict of interest exists.

ed that APOC3 more strongly predicted CAD than TG. Together, the results of this study remind us of the longstanding (and continuing) battle between supporters of HDL cholesterol and supporters of TG as independent causal cardiovascular disease (CVD) predictors (3) and suggest that future experiments should probe the predictive values of APOB or measured TG-rich-lipoprotein (TRL) cholesterol in the CACTI cohort (4).

APOC3 levels are elevated in individuals with T1DM; however, the drivers of this increase are not fully understood. Twenty-five years ago, Chen and colleagues reported that insulin binding to an "insulin response element" inhibited APOC3 gene expression (5). Later studies showed that FoxO1 mediates insulin action on the APOC3 promoter (6). Hyperglycemia, independent of insulin, was identified as an additional APOC3 transcriptional activator, acting through ChREBP and HNF4 $\alpha$ (7). Kanter et al., however, present convincing evidence via mouse models that insulin-mediated alterations of circulating APOC3 levels are the result of posttranscriptional effects, rather than changes in transcription. Moreover, the authors suggest that the reductions in APOC3 observed during intensive insulin treatment resulted from improved uptake of remnant lipoproteins (RLPs) by the liver (2). Whereas this hypothesis needs to be tested, APOC3 secretion rates have been measured in humans using stable isotopes and are increased in insulin-resistant individuals with high TG levels (8) and in patients with type 2 diabetes (9). Additionally, we demonstrated previously that treatment of individuals with type 2 diabetes with pioglitazone, a PPAR $\gamma$ agonist and insulin sensitizer, reduced secretion rates of APOC3 (10). Thus, the basis for elevated plasma levels of APOC3 may vary by the model used and by disease state.

Most importantly, why are elevations in APOC3 associated with dyslipidemia and increased CVD in humans as well 
as the mouse models? It is possible that APOC3 is acting as a backseat passenger carried by TRLs and RLPs, and, as such, is a marker of an atherogenic dyslipidemia that is present because of other underlying abnormalities, including insulin resistance or diabetes mellitus. Although dyslipidemia may, to some degree, precede an increase of APOC3 levels in CVD, there is clear evidence that genetically increasing $(11,12)$ or decreasing (13-15) APOC3 results in hyper- or hypolipidemia, respectively. Thus, even if the presence of increased concentrations of TRLs and RLPs contribute to higher APOC3 levels, the role of APOC3 as an inhibitor of lipoprotein lipase-mediated (LPLmediated) lipolysis (16) and RLP uptake by the liver (17), would sustain and exacerbate the dyslipidemia. These findings suggest that for dyslipidemia, APOC3 is a passenger that is at least sitting in the front seat of the car.

\section{The antiatherogenic potential of targeting APOC3}

But could APOC3 be the actual driver of CVD? Kanter et al. used an APOC3targeting antisense oligonucleotide (ASO) to examine this question in great detail (2). Nearly complete APOC3 knockdown by the ASO markedly reduced plasma TG and VLDL cholesterol levels, as well as plasma APOE levels in diabetic mice. These changes in the number and composition of TRLs and RLPs prevented development of CVD in diabetic mice. Examination of atherosclerotic lesions revealed reduced accumulation of APOC3, APOE, APOB, and macrophages, in APOC3 ASO-treated mice. Remarkably, in a model of preexisting atherosclerosis, the APOC3 ASO reduced the size of necrotic cores. Kanter et al. also reported reductions in the number and composition of circulating TRLs and RLPs during ASO treatment that would confound interpretation of the role of APOC3. Therefore, future experiments in which the degree of dyslipidemia is maintained (i.e., concomitantly reducing LPL), while difficult to perform, are worthy of consideration.

Of note, a number of non-lipid effects of APOC3, including induction of adhesion molecules and inflammation in cultured vascular endothelial cells and vascular smooth muscle cells, have been reported $(18,19)$. These effects have been linked to increased adhesion of monocytes to cultured vascular endothelial cells (20). Although Kanter and colleagues did not address these specific "pleiotropic" effects of APOC3, they did examine the effect of the APOC3 ASO on several aspects of inflammation. Despite marked reductions in macrophage accumulation in the atherosclerotic lesion of diabetic mice during APOC3 ASO treatment, circulating leukocytes remained constant. Similarly, APOC3 ASO treatment failed to reduce inflammation in peritoneal macrophages. Conversely, two prominent players in the acute phase response of mice, serum amyloid A1 and A2 (SAA1 and SSA2), were modestly increased during APOC3 ASO treatment. The authors concluded that reductions in APOC3 did not reduce inflammation in their models.

Overall, these studies extend our understanding of a complex and sometimes contentious area of APOC3 biology. The role of APOC3 in lipoprotein metabolism is inherently complicated, making it difficult to isolate the consequences of increasing or decreasing circulating levels. We do not view APOC3 as simply a backseat passenger, but as a molecule that, if not actually driving, is in the front seat riding shotgun, giving precise directions to the driver at intersections and curves in the road. Despite the absence of a complete understanding as to why increased APOC3 is so closely linked to proatherogenic alterations in plasma lipoproteins and CVD, further evaluation of the antiatherogenic potential of reducing APOC3 concentrations in all people at high risk for CVD will be a worthy endeavor.

\section{Acknowledgments}

We would like to acknowledge all of the investigators, only some of whom have been referenced here, who have, for the past 50 years, generated exciting and increasingly relevant data about the roles of APOC3 in lipid and lipoprotein metabolism. This commentary was supported by NIH grant R35 HL135833 (to HNG).

Address correspondence to: Henry N. Ginsberg, Department of Medicine, Vagelos College of Physicians and Surgeons, Columbia University, New York, New York, USA. Phone: 212.305.9562; Email: hng1@cumc.columbia.edu.
1. Dabelea D, et al. Effect of type 1 diabetes on the gender difference in coronary artery calcification: a role for insulin resistance? The Coronary Artery Calcification in Type 1 Diabetes (CACTI) Study. Diabetes. 2003;52(11):2833-2839.

2. Kanter JE, et al. Increased apolipoprotein C3 drives cardiovascular risk in type 1 diabetes. J Clin Invest. 2019;129(10):4165-4179.

3. Emerging Risk Factors Collaboration, et al. Lipid-related markers and cardiovascular disease prediction. JAMA. 2012;307(23):2499-2506.

4. Sniderman AD, et al. Hypertriglyceridemia and cardiovascular risk: a cautionary note about metabolic confounding. J Lipid Res. 2018;59(7):1266-1275.

5. Chen M, Breslow JL, Li W, Leff T. Transcriptional regulation of the apoC-III gene by insulin in diabetic mice: correlation with changes in plasma triglyceride levels. J Lipid Res. 1994;35(11):1918-1924

6. Altomonte J, et al. FoxO1 mediates insulin action on apoC-III and triglyceride metabolism. J Clin Invest. 2004;114(10):1493-1503.

7. Caron S, et al. Transcriptional activation of apolipoprotein CIII expression by glucose may contribute to diabetic dyslipidemia. Arterioscler Thromb Vasc Biol. 2011;31(3):513-519.

8. Cohn JS, Patterson BW, Uffelman KD, Davignon J, Steiner G. Rate of production of plasma and very-low-density lipoprotein (VLDL) apolipoprotein C-III is strongly related to the concentration and level of production of VLDL triglyceride in male subjects with different body weights and levels of insulin sensitivity. JClin Endocrinol Metab. 2004;89(8):3949-3955.

9. Adiels M, et al. Role of apolipoprotein C-III overproduction in diabetic dyslipidaemia. Diabetes Obes Metab. 2019;21(8):1861-1870.

10. Nagashima K, et al. Effects of the PPARgamma agonist pioglitazone on lipoprotein metabolism in patients with type 2 diabetes mellitus. J Clin Invest. 2005;115(5):1323-1332.

11. Ito Y, Azrolan N, O'Connell A, Walsh A, Breslow JL. Hypertriglyceridemia as a result of human apo CIII gene expression in transgenic mice. Science. 1990;249(4970):790-793.

12. Masucci-Magoulas L, et al. A mouse model with features of familial combined hyperlipidemia. Science. 1997;275(5298):391-394.

13. Maeda N, Li H, Lee D, Oliver P, Quarfordt $\mathrm{SH}$, Osada J. Targeted disruption of the apolipoprotein C-III gene in mice results in hypotriglyceridemia and protection from postprandial hypertriglyceridemia. J Biol Chem. 1994;269(38):23610-23616.

14. Pollin TI, et al. A null mutation in human APOC3 confers a favorable plasma lipid profile and apparent cardioprotection. Science. 2008;322(5908):1702-1705.

15. Ginsberg HN, et al. Apolipoprotein B metabolism in subjects with deficiency of apolipoproteins CIII and AI. Evidence that apolipoprotein CIII inhibits catabolism of triglyceride-rich lipoproteins by lipoprotein lipase in vivo. J Clin Invest. 1986;78(5):1287-1295.

16. Brown WV, Baginsky ML. Inhibition of lipopro- 
tein lipase by an apoprotein of human very low density lipoprotein. Biochem Biophys Res Commun. 1972;46(2):375-382.

17. Windler E, Chao Y, Havel RJ. Regulation of the hepatic uptake of triglyceride-rich lipoproteins in the rat. Opposing effects of homologous apolipoprotein $\mathrm{E}$ and individual $\mathrm{C}$ apoproteins. J Biol Chem. 1980;255(17):8303-8307.
18. Yue Y, et al. RNA-seq analysis provide new insights into mapk signaling of apolipoproteinciii-induced inflammation in porcine vascular endothelial cells. Cell Cycle. 2017;16(22):2230-2238.

19. Li H, et al. Aggravated restenosis and atherogenesis in ApoCIII transgenic mice but lack of protection in ApoCIII knockouts: the effect of authentic triglyceride-rich lipoproteins with and without ApoCIII. Cardiovasc Res. 2015;107(4):579-589.

20. Kawakami A, Aikawa M, Libby P, Alcaide P, Luscinskas FW, Sacks FM. Apolipoprotein CIII in apolipoprotein B lipoproteins enhances the adhesion of human monocytic cells to endothelial cells. Circulation. 2006;113(5):691-700. 